\title{
MODELING OF HIGH ENERGY IMPACT ON ICE IN TAKING INTO ACCOUNT THE TEMPERATURE
}

\author{
Gennady N. Bogomolov ${ }^{1, *}$, Victor P. Glazyrin ${ }^{1}$, and Maxim Yu. Orlov ${ }^{1}$ \\ ${ }^{1}$ National Research Tomsk State University, 634050 Tomsk, Russia
}

\begin{abstract}
In this paper, the problems deep penetration of compact impactors into the ice, taking into account different temperatures were solved. The behavior of ice is described by the basic system equations of continuum mechanics, i.e., the equations of continuity, momentum and energy in the lagrangian approach. Medium are compressible, isotropic, no mass forces, internal sources of heat and thermal conductivity. Medium also includethe shockwave phenomena, as well as formation "spall" and "shear" damage. The stress tensor is divided into deviatoric and spherical components. Equation of statewas chosen in the form of Walsh. The components of the stress tensor deviator located on the elasticplastic flow model based on the equations of Prandtl-Reis associated with von Mises yield criterion. Initial impactor velosity was varied atfrom 50 to $325 \mathrm{~m} / \mathrm{s}$. Numerical simulation results showed the influence of temperature of the ice to the depth of penetration of the impactors.
\end{abstract}

\section{Introduction}

The urgency of research on highenergy impact on the ice in order to deepen scientific knowledge of the physics and mechanics of ice due to the intensive development is currently of the Arctic and the northern areas of Russia. In this case numerical simulatingis an effective tool to obtain detailed information about loading processes, significantly adding to the experimental results in a wide range of initial conditions.

Theoretical results on the investigation of the ice impulse load under a certain fixed temperature were obtained $[1,2]$. However, in terrestrial conditions the ice can be kept at a temperature of $0{ }^{0} \mathrm{C}$ and up to $-60{ }^{0} \mathrm{C}$. Therefore, the mathematical model of the behavior of ice should be taken into account the dependence of the strength of physical and mechanical characteristics of the temperature.

In the present research the shock process of cylindrical and spherical impactors with an ice barrier was investigated. The subsonic range of initial velocity of impactors was studied.

*Corresponding author: glvp@list.ru 


\section{Mathematical model}

The solution of the problem of phenomenological macroscopic model of continuum mechanics, which is based on elastoplastic behavior of the material. The basic system of equations is founded on the fundamental laws of conservation of mass, momentum and energy:

$$
\begin{aligned}
& \frac{1}{\rho} \frac{d \rho}{d t}=-\left(\frac{\partial u}{\partial z}+\frac{\partial v}{\partial r}+\frac{v}{r}\right) \\
& \rho \frac{d u}{d t}=\frac{\partial S_{z z}}{\partial z}+\frac{\partial S_{r z}}{\partial r}+\frac{S_{r z}}{r}-\frac{\partial P}{\partial z}, \\
& \rho \frac{d v}{d t}=\frac{\partial S_{r r}}{\partial r}+\frac{\partial S_{r z}}{\partial z}+\frac{S_{r r}-S_{\theta \theta}}{r}-\frac{\partial P}{\partial r}, \\
& \frac{d \varepsilon}{d t}=\frac{P}{\rho^{2}} \frac{d \rho}{d t}+\frac{1}{\rho}\left[S_{z z} \frac{\partial u}{\partial z}+S_{r r} \frac{\partial v}{\partial r}+S_{\theta \theta} \frac{v}{r}+S_{r z}\left(\frac{\partial v}{\partial z}+\frac{\partial u}{\partial r}\right)\right] . \\
& \frac{D S_{z z}}{D t}+\lambda S_{z z}=2 \mu\left(\frac{\partial u}{\partial z}+\frac{1}{3 \rho} \frac{d \rho}{d t}\right), \frac{D S_{r r}}{D t}+\lambda S_{r r}=2 \mu\left(\frac{\partial v}{\partial r}+\frac{1}{3 \rho} \frac{d \rho}{d t}\right), \\
& \frac{d S_{\theta \theta}}{d t}+\lambda S_{\theta \theta}=2 \mu\left(\frac{v}{r}+\frac{1}{3 \rho} \frac{d \rho}{d t}\right), \frac{D S_{r z}}{D t}+\lambda S_{r z}=\mu\left(\frac{\partial u}{\partial r}+\frac{\partial v}{\partial z}\right), \\
& S_{r r}^{2}+S_{z z}^{2}+S_{r z}^{2}+S_{r r} S_{z z}=\frac{1}{3} \sigma_{m}^{2}, P=P(\rho, \varepsilon), \sigma_{i j}=S_{i j}-P \delta_{i j}, \\
& 2 \mu\left(e_{i j}-\frac{1}{3} e_{k k} \delta_{i j}\right)=\frac{D S_{i j}}{D t}+\lambda S_{i j}, \\
& \frac{D S_{i j}}{D t}=\frac{d S_{i j}}{d t}-S_{i k} w_{j k}-S_{j k} w_{i k}, \quad \sigma_{1}>\sigma_{k} \\
& 2 e_{i j}=\nabla_{j} v_{i}+\nabla_{i} v_{j} ; \quad 2 w_{i j}=\nabla_{j} v_{i}-\nabla_{i} v_{j} \text {; } \\
& 3 S_{i j} S_{i j}=2 \sigma_{T}^{2} \text {. } \\
& A_{p} \geq A_{P}^{*}, \quad A_{p}=\int_{0}^{t_{*}} d A_{p}=\int_{0}^{t_{*}} \frac{\sigma_{i j} d \varepsilon_{i j}^{p}}{\rho},
\end{aligned}
$$

where are $\rho$-density of the material; $r, z$ - radial and axial coordinates; $u, v$-axial and radial components of the velocity vector; $\sigma_{i j}, \varepsilon_{i j}, e_{i j}$ - tensor components of stress, strain and strain rate; $\varepsilon$ - specific internal energy, $\delta_{i j}$ - Kronecker symbol,$P$ - hydrostatic pressure; $\mu$ - shear modulus; $\sigma_{T}$ - yield strength of the material; $\sigma_{\mathrm{k}}$ - "spall" damage; $\mathrm{A}_{\mathrm{p}}$ compressive shear plastic deformation; D/Dt- derivative of Yaumana. Yauman derivative take into account medium elements rotate while it is moving.

The strength of ice characteristics depend on the temperature as follows:

$$
f_{i}=f_{i 0}\left(1+\frac{1}{f_{i 0}} \frac{\partial f}{\partial t} \Delta T\right)=f_{i 0}+a_{i} \Delta T,
$$


where $f_{i}$ - shear modulus, yield stress, tension spall and critical work of plastic deformation; $\mathrm{f}_{\mathrm{i} 0}$ - initial values of these characteristics. Constants $a_{i}$ determined from experimental data $[3,4,5]$ and the condition of the vanishing ice at zero temperature. The following values of strength characteristics used in the calculations:

$\mu_{0}=3.1 \mathrm{MPa} ; \sigma_{\text {то }}=1.85 \mathrm{MPa} ; \sigma_{\mathrm{ko}}=3.06 \mathrm{MPa} ; \mathrm{A}_{\mathrm{o}}=0.3 \mathrm{KJ} / \mathrm{kg} ; \mathrm{a}_{1}=0.035 \mathrm{MPa} /$ degree;

$\mathrm{a}_{2}=0.030 \mathrm{MPa} /$ degree; $\mathrm{a}_{3}=0.030 \mathrm{MPa} /$ degree; $\mathrm{a}_{4}=0.02 \mathrm{KJ} /(\mathrm{kg} \times \mathrm{deg})$.

\section{Numerical simulation}

Physical formulation of the problems is following. The cylinder and the sphere of the same mass penetrate into ice barrier. Initial velocity impactors directed along its axis of symmetry normal to the plane of the barrier and was set equal to 50 to $325 \mathrm{~m} / \mathrm{s}$. The object of study is the ice cylinder. Cylinder height is $60 \mathrm{~mm}$, but diameter is $45 \mathrm{~mm}$.

There were 12 of calculation variants. In each variant the initial velocity of the impactor increased to $25 \mathrm{~m} / \mathrm{s}$. The cylinder has of dimensions $(5 \times 5) \mathrm{mm}$. Diameter of the spherical impactor is equal to $5.72 \mathrm{~mm}$. Impactors weight are $0.77 \mathrm{~g}$. The boundary conditions at the contact surfaces allow their sliding without friction. Material of impactors were steel U10A.

The initial conditions for problems of shock interaction of solids for the undisturbed state imply the vanishing stress component, pressure and internal energy. The numerical solution of the boundary value problem is carried out in a two-dimensional statement for the axial symmetry of themodified method Johnson G.R. It is known that this method is a lagrangian, but it allows solve the problem of deep penetration of heterogeneous structure, including modern safety structure. For this incorporated the algorithm erosion of triangulation elements and algorithm splitting nodes, the algorithm for constructing the free surface $[6,7]$. The latest innovation should be considered smoothing algorithm for the contact surface [8].

For a plane and axial symmetry calculation program has been developed in a twodimensional formulation that allows you to prepare online original data, including automatic partitioning of the computational domain. The computer program allows the application of five different equations of state for many materials of construction [8].

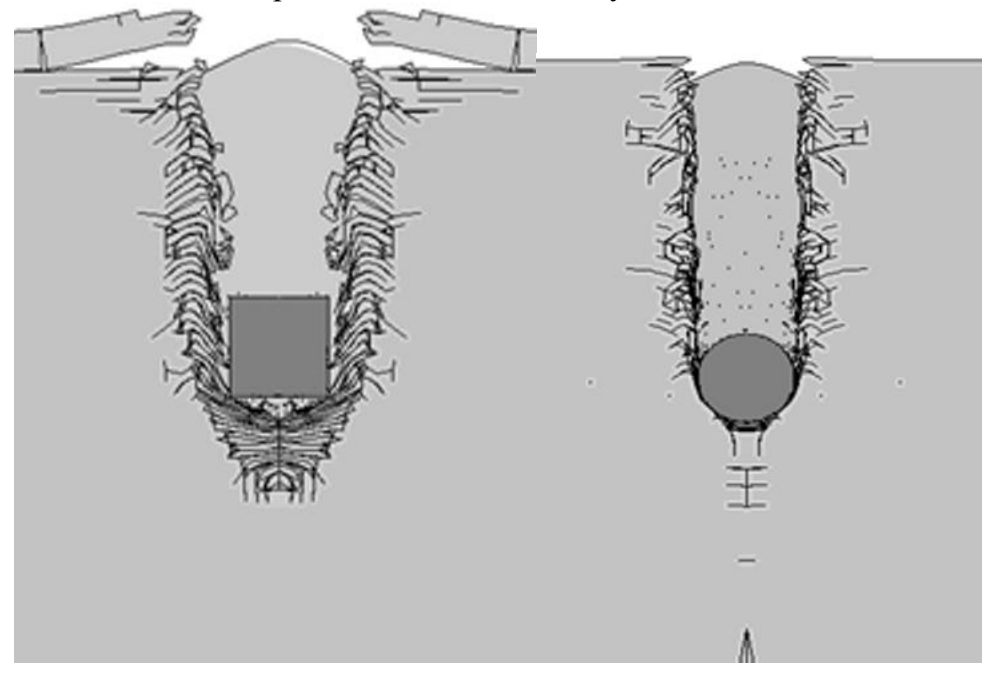

Fig. 1. Current configurations "Impactor - Ice", time 225 mks. 


\section{Results}

The calculated configurations of "Impactor - Ice" are shown in figure 1. The initial velocity of the impactors are $225 \mathrm{~m} / \mathrm{s}$. It was found that the first destruction of the pockets formed in the ice in the contact zone "impactor - ice" at the begining process. The deeply penetrated impactors into the ice, the greater the area of destruction of ice was. The deformation of the cylindrical and spherical impactors occurs in the elastic field without failure impactors. The penetration of the cylindrical impactor accompanied by spall fracture front surface of the ice barriers. The depth of penetration of the spherical impactor was 15 $\%$ more than the depth of penetration of the cylindrical impactor. Obviously, this is explained streamline shape of the spherical impactor.

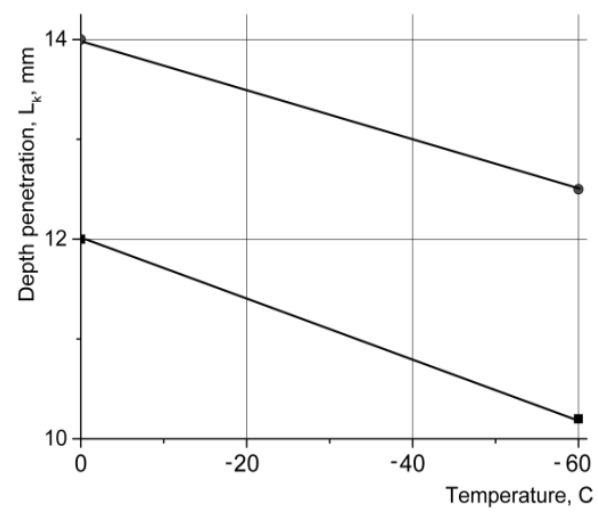

Fig. 2. Calculated dependence the depth of penetration of the impactors from ice temperature (• - spherical impactor, $\square$ - cylindrical impactor).

Figure 2 illustrates the dependence of penetration depth of impactors on the temperature. The upper line corresponds to the dependence of the spherical the impactor on the temperature and the bottom line corresponds to dependence of the cylindrical the impactor on the temperature.In this variant the initial velocity of impactors was equal to $175 \mathrm{~m} / \mathrm{s}$. The temperature changed from 0 to $-60^{\circ} \mathrm{C}$. It was found that with an increase in negative ice temperature depth penetration of impactors decreased in almost a linear relationship. It was found that the penetration of the cylindrical the impactor into ice decrease in depth of penetration was $16.3 \%$. At the same time the penetration of the spherical the impactor into ice decrease the depth of penetration was $15.2 \%$. In both cases, the impactors remained undamaged.

\section{Conclusions}

Thus the process penetration impactors into ice at low impact velocity have been simulated. Ice modeled by a single-phase medium with different negative temperature. Current and final parameters of the process, as well as identify areas destroyed by ice from the magistral crack and the first foci of destruction were predicted. The calculation results can be useful in the construction of approximate formulas for express analysis of the relevant bodies of shock interaction. 


\section{Acknowledgments}

The work was supported by the RF Ministry of Education as part of the state task No. 2014 / 223 (project 1567).

\section{References}

1. V.P. Glazyrin, Ju.N. Orlov, Ju.N. Orlova, Izvestija vuzov. Fizika 52, 7 (2009)

2. A. Combescure, Y. Cyuzel-Marmot, J. Fabis, Int. J. Solids Structures 48 (2011)

3. V.V. Bogorodskij, V.P. Gavrilo, Led. Fizicheskie svojstva. Sovremennye metody gljaciologii (1980)

4. K.S. Carney et. al., Int. J. Solids Structures 43 (2006)

5. R.G. Kraus et. al., Earth and Planetary Science Letters 289 (2010)

6. V.P. Glazyrin, M.Ju. Orlov, Ju.N. Orlov, Izvestija vuzov. Fizika 50, 9 (2007)

7. A.V. Gerasimov, V.P. Glazyrin, V.N. Barashkov $i$ dr. Teoreticheskie $i$ jeksperimental'nye issledovanija vysokoskorostnogo vzaimodejstvija tel, (2007)

8. V.P. Glazyrin, Ju.N. Orlov, Research Journal of International Studies 11, 42 (2015) 